\title{
Limiting Link Disclosure in Social Network Analysis through Subgraph-Wise Perturbation
}

\author{
Amin Milani Fard \\ School of Computing Science \\ Simon Fraser University \\ Burnaby, BC, Canada \\ milanifard@cs.sfu.ca
}

\author{
Ke Wang \\ School of Computing Science \\ Simon Fraser University \\ Burnaby, BC, Canada \\ wangk@cs.sfu.ca
}

\author{
Philip S. Yu \\ Dept. of Computer Science \\ University of Illinois at Chicago \\ Chicago, Illinois, USA \\ psyu@cs.uic.edu
}

\begin{abstract}
Link disclosure between two individuals in a social network could be a privacy breach. To limit link disclosure, previous works modeled a social network as an undirected graph and randomized a link over the entire domain of links, which leads to considerable structural distortion to the graph. In this work, we address this issue in two steps. First, we model a social network as a directed graph and randomize the destination of a link while keeping the source of a link intact. The randomization ensures that, if the prior belief about the destination of a link is bounded by some threshold, the posterior belief, given the published graph, is no more than another threshold. Then, we further reduce structural distortion by a subgraph-wise perturbation in which the given graph is partitioned into several subgraphs and randomization of destination nodes is performed within each subgraph. The benefit of subgraph-wise perturbation is that it retains a destination node with a higher retention probability and replaces a destination node with a node from a local neighborhood. We study the trade-off of utility and privacy of subgraph-wise perturbation.
\end{abstract}

\section{Categories and Subject Descriptors}

H.2.8 [Database Applications]: Data Mining; K.4.1 [Public Policy Issues]: Privacyc

\section{General Terms}

Algorithms, Measurement, Design, Experimentation

\section{Keywords}

Social networks, Privacy-preserving data publishing, Graph node partitioning

\section{INTRODUCTION}

Social network analysis is gaining more attention in the study of many human and natural phenomena. However, publishing the structure of a social network for such analysis

Permission to make digital or hard copies of all or part of this work for personal or classroom use is granted without fee provided that copies are not made or distributed for profit or commercial advantage and that copies bear this notice and the full citation on the first page. To copy otherwise, to republish, to post on servers or to redistribute to lists, requires prior specific permission and/or a fee.

EDBT 2012, March 26-30, 2012 Berlin, Germany

Copyright 2012 ACM 978-1-4503-0790-1/12/03 ...\$10.00. may reveal sensitive information about participants. A compromise is often reached between the data publisher and the data miner, resulting in both parties agreeing to some methods that will be used to anonymize the network data prior to its publication. Graph anonymization is more challenging than relational data because the adversary's background knowledge can be any information about nodes, links, subgraphs, and etc. [3]. Another challenge is that measuring information loss of graph anonymization can be tricky because two graphs with the same number of vertices and the same number of links can vary considerably in their global properties such as betweenness, diameter, etc., that are central to social network analysis [17].

Several types of privacy disclosure for social network data have been identified: identity disclosure, link disclosure, and content disclosure [15]. Content disclosure refers to sensitive disclosure of content information associated with each individual, such as age, gender, sex orientation, etc. Such disclosures are typically addressed by data anonymization [1][11]. Even if no such content information is released, identity disclosure can occur, which refers to the re-identification of the node of a target individual using background knowledge on neighborhood structure such as the degree of a node [3]. Identity disclosure is typically limited by node anonymization, which renders the neighborhood of several nodes similar. Such techniques include degree $k$-anonymity [4][15][23], $k$-automorphism [24], and $k$-isomorphism [9]. Link disclosure refers to inference about the existence of a link between two known individuals and is the focus of this work.

\subsection{Motivation}

In this work, we consider the problem of limiting link disclosure, that is, we want to limit the ability of an adversary to infer the existence of a link between two individuals. The work in [22] shows that knowledge about the links of a node can undo node anonymization. In this sense, limiting link disclosure is more fundamental than limiting identity disclosure. One powerful technique for limiting link disclosure is link perturbation [13][18], where some number of randomly selected links are deleted from the graph and some number of randomly selected new links are inserted into the graph. The number of links deleted and the number of links inserted dictate the trade-off between utility and privacy. The analysis in [6] show that link perturbation may achieve meaningful levels of identity obfuscation while still preserving characteristics of the original graph.

All previous works on link perturbation have the following characteristics (or limitations). 
- First, despite the fact that many real life social networks (e.g., Twitter followers, e-mail networks, Google+ circles, Facebook) are directed graphs, previous works in the field of privacy preserving data publishing model a social network as an undirected graph. For example, a directed link $(u, v)$ in a trust network means that $u$ trusts (or distrusts) $v$, whereas a link $(v, u)$ means that $v$ trusts (or distrusts) $u$. The privacy implication of disclosing the two links is different. Dealing with only undirected graphs, previous link perturbation [13][18] will randomize both the source node $u$ and the distinction node $v$ of a link $(u, v)$, even though, to hide the link $(u, v)$, it suffices to hide either the source $u$ or the destination $v$. Consequently, considerable structural distortion is incurred.

- Second, the prior belief of a destination node is not factored in inferring a destination node. The long-tail degree distribution of social networks implies that a small number of nodes are highly popular destination nodes of links [5]. Hiding the links to such celebrity nodes is more difficult or even unrealistic because of the prior knowledge due to such a popularity. A reasonable privacy notion should take this prior into account and protect a destination node only if the prior is bounded by some threshold.

- Third, the deleted/inserted links are selected randomly from the entire graph without considering the structural proximity of nodes. For example, adding a link between two nodes that are far apart would make the graph useless for shortest path analysis, or deleting the only link connecting two parts of the graph would make the two parts disconnected.

\subsection{Contributions}

To address the above issues, we propose several important deviations from previous works. Our contributions are summarized as follows.

Contribution 1 We model a social network as a directed graph and hide the existence of a link by perturbing the destination node of the link while keeping the source node intact. Specifically, for each (directed) link $(u, v)$, we retain $(u, v)$ with a certain probability $p$ and replace $(u, v)$ with a link $(u, w)$ with probability $1-p$, where $w$ is randomly selected from all destination nodes in the graph. We name this solution graph-wise perturbation. Note that being able to handle directed graphs is a generalization, not a restriction, because undirected graphs can always be modeled by directed ones.

Contribution 2 We formalize the privacy of a link through $\left(\rho_{1}, \rho_{2}\right)$-privacy proposed in [10]. Informally, $\left(\rho_{1}, \rho_{2}\right)$-privacy, where $0<\rho_{1}<\rho_{2}<1$, says that if the adversary's prior belief (before seeing the published graph) that a node $v$ is the destination of a link is no more than $\rho_{1}$, his posterior belief (after seeing the perturbed graph) that $v$ is the true destination of a link is no more than $\rho_{2}$. In other words, publishing the data changes the belief of the adversary by at most $\rho_{2}-\rho_{1}$. We give the maximum retention probability $p$ for satisfying a given $\left(\rho_{1}, \rho_{2}\right)$-privacy requirement.

Contribution 3 We propose subgraph-wise perturbation in which the given graph is partitioned into local subgraphs and graph-wise perturbation is applied to each subgraph. By confining the choice of a randomized node to a smaller subgraph, this approach is able to preserve more structure of the graph by retaining a destination node of a link with a higher retention probability and by replacing a destination node with a node from a local neighborhood. We present an analysis on the impact of graph partitioning on privacy, and a way to reduce the impact. We study empirically the trade-off between utility and privacy.

The rest of the paper is organized as follows. Related work, preliminaries, and problem description are presented in Section 2, 3, and 4. Our subgraph-wise perturbation, experiments, and conclusion appear in Section 5, 6, and 7 .

\section{RELATED WORK}

Most previous works consider limiting identity disclosure, e.g., [4][15][23][24]. As shown in [9], limiting identity disclosure does not limit link disclosure. Our work is most related to link disclosure. The first group of works in this area achieves some form of edge anonymity by transforming the graph to have some structural similarity. $k$-isomorphism [9] is a way to achieve such structural similarity where the original graph is transformed into $k$ disconnected pairwise isomorphic subgraphs through link insertion and deletion. Another approach is partitioning by nodes into equivalence classes and inducing edge equivalence classes grouping links between node classes[20]. In general, considerable structural distortion is required to provide the desired structural similarity.

The second group of works is based on random link perturbation. The work in [18][13] considered randomly adding $m$ non-existing links, randomly deleting $m$ existing links, or randomly switching $m$ pairs of links (by switching their endpoints). As explained earlier, since the graph is considered undirected and since the deleted links and inserted links are chosen from the entire graph, such perturbation incurs considerable structural distortion.

The third group of works aims to hide the existence of links from an adversary who employs link prediction techniques to infer the presence of sensitive links from nonsensitive links. [21] is a work in this group. To publish a graph, their method removes all sensitive links and some non-sensitive links in order to limit the adversary's ability of predicting (removed) sensitive links. We assume that all links in a graph are sensitive; therefore, if their method is applied to our graphs, no link will be published.

To our knowledge, our work is the first that models a social network as a directed graph in the field of privacy preserving data publishing, adopts $\left(\rho_{1}, \rho_{2}\right)$-privacy [10] to factor in the prior belief of a destination node, and retains more structure of a graph by subgraph-wise perturbation. Note that [10] does not consider social network data or link privacy.

\section{PRELIMINARIES}

In this section we introduce our data model, assumptions, perturbation operator, and privacy/utility measures.

\subsection{Social Network Data}

We represent a social network by a simple directed graph $G=(V, E)$, where $V$ is the set of nodes $\{1, \ldots,|V|\}$ representing users, and $E$ is the link table on two columns $(N 1, N 2)$ and contains one row for each link $(u, v)$ representing a relationship from user $u$ to user $v . u$ is the source 
node of the link and $v$ is the destination node of the link. Each node is associated with a unique (pseudonym) node ID and no content information about a node will be published. $\operatorname{Src}(G)$ denotes the set of all source nodes and $\operatorname{Dst}(G)$ denotes the set of all destination nodes in $G$. $d^{i n}(u)$ denotes the $i n$-degree of $u$, i.e., the number of links with $u$ being the destination node, and $d^{\text {out }}(u)$ denotes the out-degree of $u$, i.e., the number of links with $u$ being the source node. An undirected graph can be represented by replacing each undirected link $(u, v)$ with two directed links $(u, v)$ and $(v, u)$. Figure 1(a) shows the link table $E$ for the graph $G$ in Figure $1(\mathrm{c})$.

Assumptions. (1) We assume that the destination of a link in $G$ is chosen independently at random according to some underlying probability distribution (such as the power law of in-degree). This assumption is reasonable because no content information of a node is released, thus, can be used to correlate nodes. The underlying probability distribution, in the form of the in-degree distribution of nodes $\operatorname{Pr}[X=x]$, is not private, and in fact, the data miner is allowed to learn it (more details in Section 3.3 and 3.4). (2) An adversary has access to the published graph and the algorithm and parameters used to produce the published graph, including the retention probability for perturbing the destination node of a link. With this information and the independence assumption in (1), the adversary tries to infer the true destination node of a link in the original graph, by computing the posterior probability of the true destination, given that the link is observed in the published graph (which was randomized by our algorithm). We do not assume that an adversary never identifies the node of a target individual; in fact, making this assumption would result in a weak privacy model. Rather, our privacy goal is to hide the existence of a link between two individuals by bounding the posterior probability of inferring the true destination of an observed link.

\subsection{Link Perturbation}

To hide the presence of a link $(u, v)$, it suffices to hide either the source $u$ or the destination $v$ because knowing one but not the other does not reveal the existence of the link $(u, v)$. We consider hiding the destination node, but the same method can be used to hide the source node by first reversing the direction of each link.

Specifically, for each link $(u, v) \in E$, we retain the destination $v$ with some retention probability $p$ and perturb the destination $v$ to a node $w$ randomly chosen from $D s t(G)$ with the probability $\frac{1-p}{m}$, where $m=|D s t(G)|$. Note that this perturbation process is done for each link independently. These probabilities can be represented by a perturbation matrix $P_{m \times m}$ : the $i^{t h}$ column represents probabilities $P_{j i}$ that the original destination node $i$ is perturbed to a new destination node $j . P_{j i}$ is defined as:

$$
P_{j i}= \begin{cases}p+(1-p) / m & \text { if } j=i(\text { retain } i) \\ (1-p) / m & \text { if } j \neq i(\text { perturb } i \text { to } j)\end{cases}
$$

Let $G^{*}$ denote the perturbed version of $G$ and let $E^{*}$ denote the link table for $G^{*}$. Note that this perturbation operator always preserves the out-degree of a node because the source node of a link is never perturbed. Figure 1(b) shows the perturbed table $E^{*}$ with perturbed values in shaded cells, and Figure 1(d) shows the perturbed graph $G^{*}$.

During the perturbation process, duplicate links and selfloops may be generated. Such links are called singular links

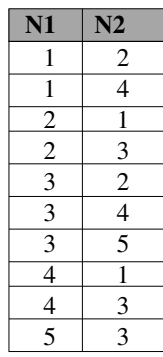

(a) Table $E$

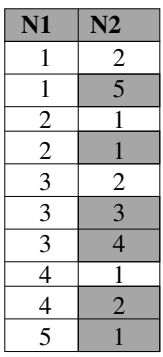

(b) Table $E^{*}$

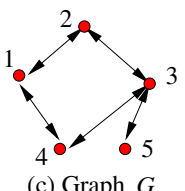

(c) Graph $G$

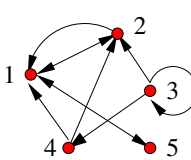

(d) Graph $G^{*}$
Figure 1: A simple social network graph

because they do not occur in the original graph $G$. We will keep all singular links because they are part of the perturbation and are useful for reconstructing the degree distribution (Section 3.4) and performing social network analysis (Section 6). We will discuss the privacy implication of such links and ways to deal with them in Section 5.3.

\subsection{Privacy Model}

We want to perturb the destination of a link to achieve some uncertainty of inferring the true destination of a published link. This goal is not achievable if a destination node is overly popular among the links. Therefore, privacy protection should be relative to the "prior" of a node as a destination of links. To capture such a relative notion of privacy, we adapt the $\left(\rho_{1}, \rho_{2}\right)$-privacy originally proposed by Evfimievski el al [10]. Consider the link table $E(N 1, N 2)$ for $G$ and the link table $E^{*}(N 1, N 2)$ for $G^{*}$. Let $r^{*}$ in $E^{*}$ be the corresponding link of a link $r$ in $E$. $X$ and $Y$ denote the random variables for the destination node $x$ in $r[N 2]$ and $y$ in $r^{*}[N 2]$ respectively. $\operatorname{Pr}[X=x]$ denotes the prior probability that $x$ is the destination of a link in $G$.

In the absence of further knowledge, we equate $\operatorname{Pr}[X=x]$ with the fraction of source nodes linking to $x$, i.e., $\frac{d^{i n}(x)}{|\operatorname{Src}(G)|}$, where $d^{i n}(x)$ is the in-degree of $x$ in $G . \operatorname{Pr}[X=x \mid Y=y]$ denotes the posterior probability that, given that a link is observed to have the destination $y$ in $G^{*}, x$ is the true destination of the link in $G$. Since the source node of a link is never perturbed, the probability $\operatorname{Pr}[X=x \mid Y=y]$ represents the adversary's ability to infer the presence of a link between two individuals in $G$. We limit this ability by $\left(\rho_{1}, \rho_{2}\right)$-privacy [10].

Definition 1. $\left(\left(\rho_{1}, \rho_{2}\right)\right.$-privacy $):$ Let $0<\rho_{1}<\rho_{2}<1$. There is an upward $\left(\rho_{1}, \rho_{2}\right)$-privacy breach w.r.t a value $x$ for $X$ if for some $y$ for $Y, \operatorname{Pr}[X=x] \leq \rho_{1}$ and $\operatorname{Pr}[X=x \mid Y=y]>\rho_{2}$. $\left(\rho_{1}, \rho_{2}\right)$-privacy holds if upward privacy breach is eliminated. $\square$

Essentially, $\left(\rho_{1}, \rho_{2}\right)$-privacy says that whenever the prior does not exceed $\rho_{1}$, the posterior must not exceed $\rho_{2}$. For a more elaborated discussion, please see [10]. This definition is more restricted than the original definition in [10] which considers the prior $\operatorname{Pr}[Q(X)]$ and posterior $\operatorname{Pr}[Q(X) \mid Y=y]$ for a general predicate $Q(X)$ on $X$. We consider the restricted form $X=x$ of $Q(X)$ because we are concerned with the risk of inferring an individual destination node $x$ of a link.

The values of $\rho_{1}$ and $\rho_{2}$ are set by the data publisher. For a destination node $x,\left(\rho_{1}, \rho_{2}\right)$-privacy requirement imposes the bound $\rho_{2}$ on $\operatorname{Pr}[X=x \mid Y=y]$ if and only if $\operatorname{Pr}[X=x] \leq \rho_{1}$. The power-law or long-tail distribution of degrees for social 
networks suggests that a small number of nodes may have a very large degree whereas the rest have a small degree [5]. Therefore, $\left(\rho_{1}, \rho_{2}\right)$-privacy will bound the posterior by $\rho_{2}$ for the majority of nodes, i.e., those with $\operatorname{Pr}[X=x] \leq \rho_{1}$.

For the small number of nodes with $\operatorname{Pr}[X=x]>\rho_{1}$, the prior is considered too high and link disclosure comes as a result of "prior belief", which occurs before the data release. Since such "celebrity" nodes are commonly referenced by other nodes, the existence of a link to such nodes is a "fact of life" and less sensitive to infer. Indeed, even if all links to celebrity nodes are removed from the published graph, the prior belief cannot be removed and thus no algorithm could prevent link disclosure for such nodes. For this reason, we focus on link disclosure mainly due to the release of data, like in most previous works. With the pre-condition $\operatorname{Pr}[X=x] \leq \rho_{1}$, our privacy goal is to limit the inference of destination nodes that have a low in-degree. This in turn limits the adversary's ability to reconstruct the original graph.

We should mention that [10] does not consider social network data or privacy notions for links. We borrow from [10] the standard notion of $\left(\rho_{1}, \rho_{2}\right)$-privacy, but all social network related contributions are new.

\subsection{Utility Metrics}

The graph for a social network is published to serve a variety of analysis purposes. We consider three types of utility metrics in this regard: (i) common graph metrics such as centrality, clustering coefficient, diameter, and etc. [17]; (ii) the destination retention probability $p$ (Equation (1)), which indicates the portion of original links preserved; (iii) the reconstruction of the in-degree of each node, which has applications such as popularity based ranking and influence of nodes. Note that our perturbation does not alter the out-degree of a node. Since (i) and (ii) are well defined, we explain only (iii).

Let $D=<d_{1}, \cdots, d_{m}>$ be the in-degree vector of destination nodes in $G$, and $O=<o_{1}, \cdots, o_{m}>$ be the observed in-degree vector of destination nodes in $G^{*}$, where $m=|D s t(G)|$. The data miner has access to $O$ but not $D$, and wants to reconstruct an estimate of $D$. Directly using $O$ as the estimate of $D$ would give a large distortion because it does not take into account of the perturbation of destination nodes. A better estimate can be obtained by treating $O$ as the result of applying the perturbation to the original graph, which is characterized by the perturbation matrix $P$ in Equation (1) (recall that $P$ is public). Let $E(O)=<E\left(o_{1}\right), \cdots, E\left(o_{m}\right)>$, where $E\left(o_{j}\right)$ is the mean of $o_{j}, \sum_{i=1}^{m} P_{j i} \times d_{i}$. We have $E(O)=P \times D$. Approximating $E(O)$ by the observed instance $O$, we get an estimate of $D$ as $D^{\prime}=P^{-1} \times O$, where $P^{-1}$ is the inversion of $P$. $D^{\prime}$ can be computed by the iterative Bayesian reconstruction [2]

Definition 2. (Reconstruction error): Let $D$ and $D^{\prime}$ be the actual and estimated in-degree vectors for $G$ and $G^{*}$ respectively. The reconstruction error is $\frac{\sum_{i}\left|d_{i}-d_{i}^{\prime}\right|}{\sum_{i} d_{i}}$.

\section{PROBLEM DESCRIPTION}

The next question is what retention probability $p$ is required to provide $\left(\rho_{1}, \rho_{2}\right)$-privacy on destination nodes. We propose two solutions, graph-wise perturbation and subgraphwise perturbation.

\subsection{Graph-wise Perturbation}

In graph-wise perturbation, the replacing destination node is randomly selected from the entire domain of the destination nodes, $\operatorname{Dst}(G)$. In this case, $m=|\operatorname{Dst}(G)|$ for the perturbation matrix $P$ in Equation (1). We can determine the maximum retention probability $p$ in Equation (1) for ensuring $\left(\rho_{1}, \rho_{2}\right)$-privacy from a constraint called amplification condition due to [10]. Intuitively, this condition says that for any two destination nodes $k$ and $i$, their probabilities of being perturbed to the same node $j$, i.e., $P_{j k}$ and $P_{j i}$, must not differ by a factor of more than $\gamma$. Precisely, let $X$ be a variable for a true destination node of a link in $G$, and $Y$ be a variable for an observed destination node of a link in $G^{*}$. A perturbation matrix $P$ is said to satisfy the $\gamma$-amplification condition [10] for a value $j$ of $Y$ if for all values $k, i$ of $X$

$$
\frac{P_{j k}}{P_{j i}} \leq \gamma
$$

Therefore, when this condition holds, the small difference between $P_{j k}$ and $P_{j i}$ implies uncertainty that both $k$ and $i$ are likely to be the original destination node, when observing a destination node $j$ in a link in the perturbed graph. The next theorem, also due to [10], relates the $\gamma$-amplification condition to $\left(\rho_{1}, \rho_{2}\right)$-privacy.

THEOREM 1. $\left(\rho_{1}, \rho_{2}\right)$-privacy is guaranteed if the perturbation matrix $P$ satisfies the $\gamma$-amplification condition for all values $j$ of $Y$, where $\gamma \leq \frac{\rho_{2}}{\rho_{1}} \times \frac{1-\rho_{1}}{1-\rho_{2}}$.

The proof of Theorem 1 can be found in [10]. Let us derive the maximum retention probability for guaranteing $\left(\rho_{1}, \rho_{2}\right)$-privacy from Theorem 1. Following Equation (1), $P_{j i}$ is maximized if $i=j$, i.e., $p+(1-p) / m$, and $P_{j i}$ is minimized if $i \neq j$, i.e., $(1-p) / m$. So the left-hand side of Equation (2) is maximized at $\frac{p+(1-p) / m}{(1-p) / m}$, when $j=k$ and $j \neq i$, and Equation (2) reduces to $\frac{p+(1-p) / m}{(1-p) / m} \leq \gamma$. To maximize the retention probability $p$, we replace $\leq$ with $=$ in the inequality and get $p=\frac{(\gamma-1)}{(m-1+\gamma)}=\frac{1}{m /(\gamma-1)+1} \cdot p$ is maximized by the maximum value of $\gamma$, which, according to Theorem 1, is

$$
\gamma=\frac{\rho_{2}}{\rho_{1}} \times \frac{1-\rho_{1}}{1-\rho_{2}}
$$

So Equation (1) with the maximum $p$ that guarantees $\left(\rho_{1}, \rho_{2}\right)$ privacy has the form

$$
P_{j i}= \begin{cases}\gamma /(m-1+\gamma) & \text { if } j=i \\ 1 /(m-1+\gamma) & \text { if } j \neq i\end{cases}
$$

with $\gamma$ being given by Equation (3). Note that $P_{j i}$ is completely determined by $m, \rho_{1}, \rho_{2}$.

Corollary 1. The perturbation matrix given by Equations (3) and (4) provides $\left(\rho_{1}, \rho_{2}\right)$-privacy.

\subsection{Subgraph-wise Perturbation}

The above graph-wise perturbation has two limitations. First, the retention probability $p=\frac{\gamma-1}{(m-1+\gamma)}$ can be very low because $m=|\operatorname{Dst}(G)|$ is large, easily thousands or more for real life social network graphs. Second, the unrestricted choice of the replacing destination node from the entire domain $\operatorname{Dst}(G)$ means that the structure of the perturbed graph could be considerably different from the original graph. 


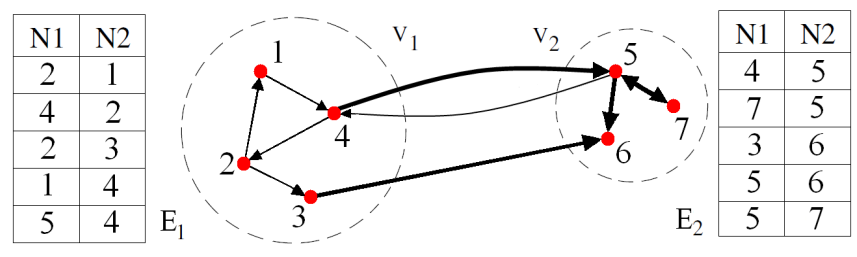

Figure 2: Link partitioning

$P^{1}=\begin{aligned} & 1 \\ & 2 \\ & 3 \\ & 4\end{aligned}\left(\begin{array}{llll}0.43 & 0.19 & 0.19 & 0.19 \\ 0.19 & 0.43 & 0.19 & 0.19 \\ 0.19 & 0.19 & 0.43 & 0.19 \\ 0.19 & 0.19 & 0.19 & 0.43\end{array}\right) \quad P^{2}=\begin{aligned} & 5 \\ & 7\end{aligned}\left(\begin{array}{lll}0.53 & 0.23 & 0.23 \\ 0.23 & 0.53 & 0.23 \\ 0.23 & 0.23 & 0.53\end{array}\right)$

Figure 3: Subgraph-wise perturbation operators

A low retention probability due to a large randomization domain was first pointed out in [8] for relational data, but their work does not address graph data and the second limitation.

To tackle these limitations, we propose a subgraph-wise perturbation approach so that the replacing destination node is chosen from some local part of the graph. First, we partition the graph $G$ into link partitioned subgraphs $G_{1}, \ldots, G_{k}$. In contrast to the usual node partitioning of a graph, the link partitioning scheme partitions the links into subgroups, so that each link in $G$ will be contained in exactly one subgraph $G_{t}$. A node may appear in multiple subgraphs and the subgraphs may not be connected. Such link partitioning ensures that, when randomizing the links in each subgraph $G_{t}$, each link in $G$ gets exactly one chance of being randomized.

Additionally, the link partitioning is expected to satisfy two properties: (i) the nodes in the same $G_{t}$ are close to each other and (ii) each subgraph $G_{t}$ has a smaller set $\operatorname{Dst}\left(G_{t}\right)$ than $\operatorname{Dst}(G)$. (i) ensures that a randomized destination node will not be structurally far away from the original destination node, and (ii) ensures a smaller domain size $m=\left|\operatorname{Dst}\left(G_{t}\right)\right|$ of destination nodes in a subgraph $G_{t}$, compared to the domain size $m=|D s t(G)|$ of destination nodes in the whole graph $G$, in order to increase the retention probability for $G_{t}$.

Example 1. Figure 2 shows a link partitioning $\left\{G_{1}, G_{2}\right\}$ and the corresponding link table partitioning $\left\{E_{1}, E_{2}\right\}$. The links in each partition are marked with a different thickness. The two circles depict destination nodes in the two partitions: $\operatorname{Dst}\left(G_{1}\right)=\left\{{ }^{\circ} 1^{\prime},{ }^{\prime} 2^{\prime},{ }^{\prime} 3^{\prime},{ }^{\prime} 4^{\prime}\right\}$ and $\operatorname{Dst}\left(G_{2}\right)=\left\{{ }^{\circ} 5^{\prime},{ }^{\circ} 6,{ }^{\prime}, 7^{\prime}\right\}$. $\square$

DeFinition 3. (Subgraph-wise perturbation): For a given $k$, we want to find a linkpartitioning of $G, G_{1}, \cdots, G_{k}$, such that, for $1 \leq t \leq k$, the nodes within each partition $G_{t}$ are close to each other and $\left|D \operatorname{st}\left(G_{t}\right)\right|$ are as small as possible. $\square$

In subgraph-wise perturbation we will randomize the links in each $G_{t}$ independently, $1 \leq t \leq k$, and produce the perturbed version $G_{t}^{*}$, using the $m_{t} \times m_{t}$ perturbation matrix $P^{t}$ defined in Equation (4), where $m_{t}=\left|\operatorname{Dst}\left(G_{t}\right)\right|$. To reconstruct the in-degree distribution $D^{\prime}$ for $G$, we can reconstruct the in-degree distribution $D_{t}^{\prime}$ from each $G_{t}^{*}$ using the method described in Section 3.4 and aggregate $D_{t}^{\prime}$ over $1 \leq t \leq k$. Subgraph-wise perturbation helps address the two limitations of graph-wise perturbation mentioned at the beginning of Section 4.2 because $m_{t}$ is smaller than $m$, where $m=|\operatorname{Dst}(G)|$, and the replacing destination node of a link in
$G_{t}$ is chosen from destination nodes in a local neighborhood, $\operatorname{Dst}\left(G_{t}\right)$. This point is illustrated by the next example.

Example 2. Consider Figure 2 again. Let $\rho_{1}=0.4$ and $\rho_{2}=0.6$, thus we have $\gamma=2.25$ (Equation (3)). With graphwise perturbation, $m=|\operatorname{Dst}(G)|=7$ and $P_{j j}=\frac{\gamma}{|D s t(G)|-1+\gamma}=$ $\frac{2.25}{7-1+2.25}=0.27$ (Equation (4)). With subgraph-wise perturbation, $\left|\operatorname{Dst}\left(G_{1}\right)\right|=4$ and $\left|\operatorname{Dst}\left(G_{2}\right)\right|=3$, and Figure 3 shows the perturbation matrixes for the subgraphs in Figure 2. $P_{j j}^{1}=\frac{\gamma}{\left|D s t\left(G_{1}\right)\right|-1+\gamma}=\frac{2.25}{4-1+2.25}=0.43$ and $P_{j j}^{2}=0.53$. This shows that the retention probabilities for the subgraphs are significantly higher than the retention probability for the original graph. $\square$

Remark 1. A question is how to publish the perturbed graphs $G_{1}^{*}, \cdots, G_{k}^{*}$. If the publication is for reconstructing the in-degree distribution of nodes, as in Section 3.4, publishing each $G_{t}^{*}$ separately will facilitate a more accurate reconstruction because each $G_{t}^{*}$ is produced by a different perturbation matrix $P^{t}$. In this case, the in-degree distribution, i.e., the prior probability, of a node in $G_{t}$, $\operatorname{Pr}_{t}[X=x]=\frac{d_{t}^{i n}(x)}{\left|\operatorname{Src}\left(G_{t}\right)\right|}$, may differ from that in the original graph $G$. We will discuss the privacy implications due to this difference in Section 5.2. In the case that the data is published for social network analysis, such as the study on closeness and betweenness centrality, clustering coefficient, graph diameter, eigenvalues, and shortest paths, it suffices to publish all $G_{t}^{*}$ in a single merged graph $G^{*}$ through common nodes without separating them apart. For example, the merged graph is obtained in Figure 2 by ignoring the different thickness of links for different partitions. In this case, the prior probability of a destination node remains unchanged.

\section{SUBGRAPH-WISE PERTURBATION}

In this section, we present an algorithm for subgraph-wise perturbation problem. This is shown in Algorithm 1. Given the input graph $G(V, E)$, the number of partitions $k$, the privacy setting $\rho_{1}$ and $\rho_{2}$, and the sparsity parameter $\ell$ (explained later), the algorithm consists of following main steps.

- Partition the node set $V$ into $k$ subsets $U_{1}, \cdots, U_{k}$ (Line 1). $U_{i}$ contains the destination nodes for subgraph $G_{i}$. This partition ensures the destinations in each $G_{i}$ are close to each.

- Induce $k$ link partitioned subgraphs $G_{1}, \cdots, G_{k}$ using $U_{1}, \cdots, U_{k}$ (Line 2). That is, $G_{i}$ contains all links to the destinations in $U_{i}$.

- Balance the in-degree of nodes in $G_{1}, \cdots, G_{k}$ to limit the prior probability of destination nodes in $G_{t}$ (Lines $3-4)$, which will be explained in Section 5.2.

- Enforce a notion of $\ell$-sparsity on each $G_{t}$ (Line 5), which will be explained in section 5.3.

- Lastly, the algorithm perturbs the destination nodes of links in each subgraph $G_{t}$ independently and outputs the perturbed $G_{t}^{*}$ and $P^{t}$ (Lines 6-11).

Steps 3 and 4 address some privacy issues introduced by the graph partitioning. The details will be discussed in Sections 5.2 and 5.3 . 


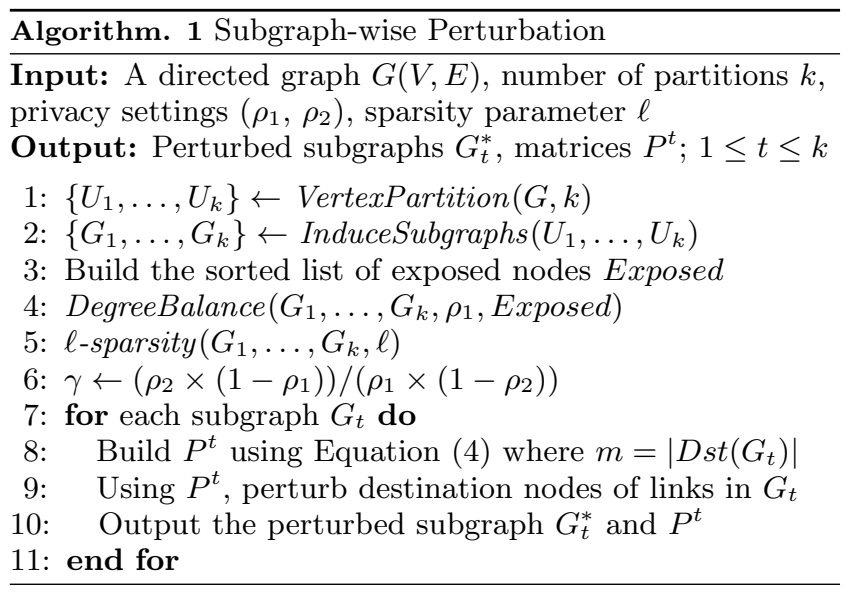

Theorem 2. Algorithm 1 is in $O(|E|+|V| \cdot \log |V|)$.

Proof. The multilevel $k$-way partitioning [14] (line 1), and inducing a link partitioning (line 2), are both in $O(|E|)$. Sorting exposed nodes (line 3 ) is in $O(|V| \cdot \log |V|)$. Degree balancing (line 4) according to Theorem 3 in Section 5.2 is in $O(|E|)$ and $\ell$-sparsity (line 5) is in $O(|V| \cdot \ell$ ). Finally generating $G^{*}$ (lines 7-11) is in $O(|E|)$. Since $\log |V|$ is typically larger than $\ell$, the overall complexity is $O(|E|+$ $|V| \cdot \log |V|)$.

Let us explain Lines 1-5 in Algorithm 1 in details.

\subsection{Link Partitioning (Lines 1-2)}

Lines 1 and 2 partition $G$ into link partitioned subgraphs $G_{1}, \cdots, G_{k}$ such that highly connected nodes are in the same subgraph and $\left|\operatorname{Dst}\left(G_{t}\right)\right|$ is as small as possible. In Line 1, VertexPartition $(G, k)$ partitions the set of nodes $V$ into $k$ disjoint sets $U_{1}, \ldots, U_{k}$ of balanced size such that the number of links with the endpoints in different sets is minimized. This is the standard node partitioned graph partitioning. We recommend $M E T I S^{1}$, an open source software for partitioning graphs [14]. Intuitively, each $U_{i}$ contains the destination nodes in the subgraph $G_{i}$. This partitioning ensures that the destination nodes in each $G_{i}$ are close to each other.

In Line 2 , InduceSubgraphs $\left(U_{1}, \ldots, U_{k}\right)$ induces a link partitioning $G_{1}\left(V_{1}, E_{1}\right), \ldots, G_{k}\left(V_{k}, E_{k}\right)$ from $U_{1}, \ldots, U_{k}$. For $1 \leq t \leq k, E_{t}$ contains all links with the destination nodes being in $U_{t}$ and $V_{t}$ is the set of nodes on either sides of the links in $E_{t}$. Since each $U_{t}$ contains highly connected nodes, the destination nodes within each $G_{t}$ are close to each other. This property is important because we want a replacing destination node to be structurally close to the original destination node. At this point, $G_{1}, \ldots, G_{k}$ is both a link partitioning and a destination-partitioning, but is not necessarily a source-partitioning, as shown in the next example. In other words, a node may appear in multiple partitions as source nodes, but appear in one partition as a destination node.

Example 3. Figure 2 shows a link partitioning $\left\{G_{1}, G_{2}\right\}$. The first step produces the node partitioning $U_{1}=\left\{{ }^{\prime} 1^{\prime},{ }^{\prime} 2^{\prime},{ }^{\prime}, 3^{\prime},{ }^{\prime} 4^{\prime}\right\}$ and $U_{2}=\left\{{ }^{\prime}{ }^{\prime},{ }^{\prime} 6,{ }^{\prime},{ }^{\prime} 7\right\}$. The second step produces the link partitioning $G_{1}\left(V_{1}, E_{1}\right)$ and $G_{2}\left(V_{2}, E_{2}\right)$, where the $i$ th partition

\footnotetext{
${ }^{1}$ http://glaros.dtc.umn.edu/gkhome/metis/metis/download
}

$E_{i}$ includes all the links that have $U_{i}$ as the destination node set. Note that a node (such as the node ' 4 ') may appear in more than one partition as a source node, but only in one partition as a destination node. $\square$

\subsection{Degree Balancing (Lines 3-4)}

$\left(\rho_{1}, \rho_{2}\right)$-privacy bounds the posterior probability $\operatorname{Pr}[X=$ $x \mid Y=y]$ only if the prior probability $\operatorname{Pr}[X=x]=\frac{d^{i n}(x)}{|\operatorname{Src}(G)|}$ is $\leq \rho_{1}$. If each $G_{t}^{*}$ is published separately, the destination node $x$ in a subgraph $G_{t}$ has a new prior $\operatorname{Pr}_{t}[X=x]=$ $\frac{d_{t}^{i n}(x)}{\left|\operatorname{Src}\left(G_{t}\right)\right|}$, where $d_{t}^{i n}(x)$ denotes the in-degree of $x$ in $G_{t}$. If $\operatorname{Pr}[X=x] \leq \rho_{1}$ but $\operatorname{Pr}_{t}[X=x]>\rho_{1},\left(\rho_{1}, \rho_{2}\right)$-privacy w.r.t. $G_{t}$ will not impose the bound $\rho_{2}$ on the posterior. We call such nodes "exposed nodes". The degree balancing step aims to reduce the number of exposed nodes.

DEFINITION 4 (EXPOSED NODES). For a destination node $x$ in $G_{t}$, if $\operatorname{Pr}[X=x] \leq \rho_{1}$ and if $\operatorname{Pr}_{t}[X=x]>\rho_{1}, x$ is called an exposed node in $G_{t}$. $\square$

Remark 2. The long-tail degree distribution of social networks implies that most nodes $x$ have a low in-degree and the prior probability $\operatorname{Pr}[X=x]$ is well below $\rho_{1}$, consequently, $\operatorname{Pr}_{t}[X=x]$ in $G_{t}$ remains below $\rho_{1}$. A few nodes that have a prior $\operatorname{Pr}[X=x]$ "close enough" to $\rho_{1}$ in $G$ may become exposed nodes after link partitioning. For example, consider the Newman's scientific collaboration network [16] with 16,264 destination nodes. Let $\rho_{1}=0.01$. The highest in-degree is 107 , thus, the highest prior probability is $\operatorname{Pr}[X=x]=\frac{107}{16,264} \leq \rho_{1}$. If we partition the graph (using $M E T I S$ ) into $k=5$ subgraphs with an (almost) equal number of destination nodes, the number of source nodes within each subgraph would be $3886,4147,4136,4481$, and 4273. Note that the partitioning is not source disjoint. To avoid becoming an exposed node, the maximum in-degree for a node in each subgraph is $\rho_{1} \times\left|\operatorname{Src}\left(G_{t}\right)\right|$, that is, 38.86, $41.47,41.36,44.81$, and 42.73 , for $G_{1}, \ldots, G_{5}$. Only 67 nodes have an in-degree above this threshold, so $\frac{67}{16,264}=0.41 \%$ of all nodes are exposed nodes.

To reduce the number of exposed nodes $x$ in $G_{t}$, the $D e$ greeBalance step reduces the prior $\operatorname{Pr}_{t}[X=x]=\frac{d_{t}^{i n}(x)}{\left|\operatorname{Src}\left(G_{t}\right)\right|}$ in two ways: either decrease $d_{t}^{i n}(x)$ by moving out some links to $x$ in $G_{t}$ to other subgraphs $G_{t^{\prime}}$ while keeping $\left|\operatorname{Src}\left(G_{t}\right)\right|$ unchanged, or increase $\left|\operatorname{Src}\left(G_{t}\right)\right|$ by moving some links from other subgraphs $G_{t^{\prime}}$ with new source nodes to $G_{t}$ while keeping $d_{t}^{i n}(x)$ unchanged. This move is called a prior-reducing move for $G_{t}$ and the subgraph $G_{t^{\prime}}$ is called the donor of the move. Additionally, such moves must not yield new exposed nodes in the donor $G_{t^{\prime}}$. We say that a prior-reducing move for $G_{t}$ involving the donor $G_{t^{\prime}}$ is safe if the move does not increase the number of exposed nodes in $G_{t^{\prime}}$.

DegreeBalance reduces the number of exposed nodes by a sequence of safe prior-reducing moves, or simply "safemoves". This is implemented by Algorithm 2. It gets an input list of exposed nodes $x$ in the ascending order of indegree, Exposed, and iteratively reduces the prior probability $\frac{d_{t}^{i n}(x)}{\left|\operatorname{Src}\left(G_{t}\right)\right|}$ by safe-moves for $G_{t}$. Exposed nodes are considered in the ascending order of in-degree because nodes with lower in-degree are more sensitive and are considered first. Each iteration has two phases. 


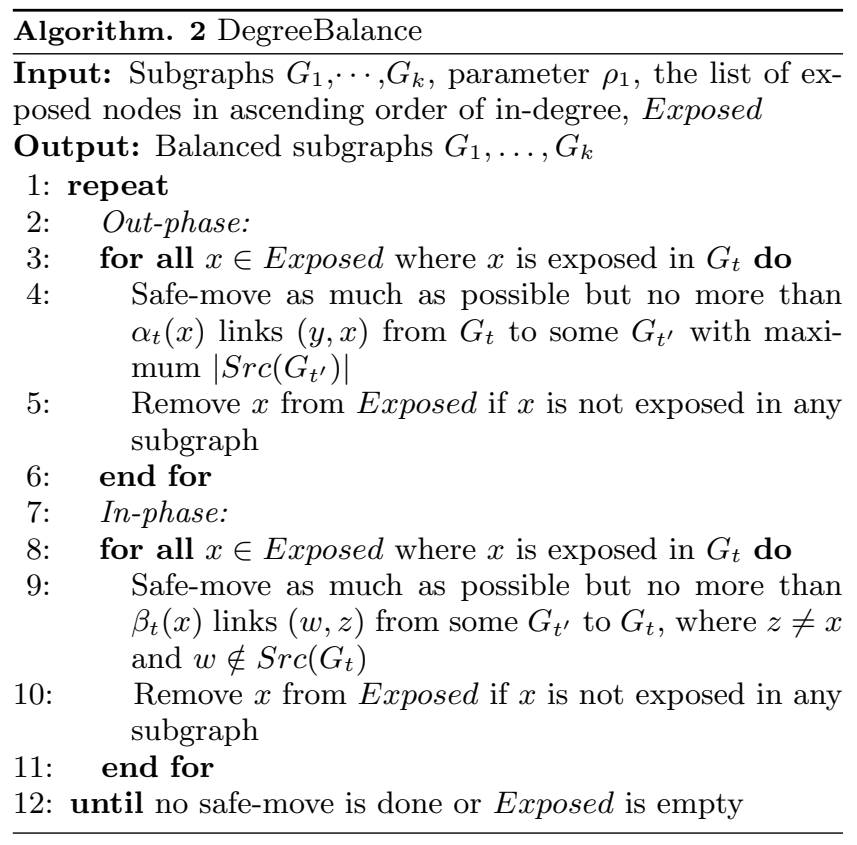

- Out-phase (line 2-6) reduces the in-degree $d_{t}^{\text {in }}(x)$ for an exposed node $x$ in $G_{t}$ by safe-moving out links to $x$ as much as possible, but no more than $\alpha_{t}(x) . \alpha_{t}(x)$ is the minimum number required to satisfy $\frac{d_{t}^{i n}(x)-\alpha_{t}(x)}{\left|\operatorname{Src}\left(G_{t}\right)\right|} \leq$ $\rho_{1}$, i.e., $\alpha_{t}(x)=\left\lceil d_{t}^{i n}(x)-\rho_{1} \times\left|\operatorname{Src}\left(G_{t}\right)\right|\right\rceil$. Given choices, we choose the donor subgraph $G_{t^{\prime}}$ with the largest $\left|\operatorname{Src}\left(G_{t^{\prime}}\right)\right|$ because such subgraphs have a small increase in the prior probability $\frac{d_{t^{\prime}}^{i n}(x)}{\left|\operatorname{Src}\left(G_{t^{\prime}}\right)\right|}$ after accepting more links to $x$.

- In-phase (line 7-11) increases $\left|\operatorname{Src}\left(G_{t}\right)\right|$ for $G_{t}$ containing some exposed nodes $x$ by safe-moving in links $(w, z)$ with new source nodes $w$ as much as possible, but no more than $\beta_{t}(x)$, which is the minimum number required to satisfy $\frac{d_{t}^{i n}(x)}{\left|\operatorname{Src}\left(G_{t}\right)\right|+\beta_{t}(x)} \leq \rho_{1} . \quad \beta_{t}(x)=$ $\left\lceil d_{t}^{i n}(x) / \rho_{1}-\left|\operatorname{Src}\left(G_{t}\right)\right|\right\rceil$.

The next theorem, shows nice properties of degree balancing in Algorithm 2.

TheOREM 3. Algorithm 2 never increases the number of exposed nodes and will terminate in $O(|E|)$.

Proof. It never increases the number of exposed nodes because it only makes safe-moves. For an exposed node $x$ in $G_{t}$, each safe-move either reduces $d_{t}^{i n}(x)$ (i.e., out-phase), or increases $\left|\operatorname{Src}\left(G_{t}\right)\right|$ (i.e., in-phase). Each such safe-move brings $\frac{d_{t}^{i n}(x)}{\left|\operatorname{Src}\left(G_{t}\right)\right|}$ closer to $\rho_{1}$, so the iterative process is guaranteed to terminate. The number of safe moves required to finish the algorithm is in $O\left(\sum_{\forall x} \alpha_{t}(x)+\sum_{\forall x} \beta_{t}(x)\right)$. Since $\sum_{\forall x} \alpha_{t}(x)$ and $\sum_{\forall x} \beta_{t}(x)$ are both in $O(|E|)$, Algorithm 2 is in $O(|\mathrm{E}|)$.

Example 4. Consider the two subgraphs in Figure 2. Let $\rho_{1}=0.4$ and $\rho_{2}=0.6 . \operatorname{Pr}_{1}\left[X={ }^{\prime} 1^{\prime}\right]=\operatorname{Pr}_{1}\left[X={ }^{\prime} 2^{\prime}\right]=\operatorname{Pr}_{1}\left[X={ }^{\prime} 3^{\prime}\right]=\frac{1}{4}$, $\operatorname{Pr}_{1}\left[X={ }^{\prime} 4^{\prime}\right]=\frac{2}{4}, \operatorname{Pr}_{2}\left[X={ }^{\prime} 5^{\prime}\right]=\operatorname{Pr}_{2}\left[X={ }^{\prime} 6^{\prime}\right]=\frac{2}{4}, \operatorname{Pr}_{2}\left[X={ }^{\prime} 7^{\prime}\right]=\frac{1}{4}$. Since the prior probability of node ' 4 ' in $G_{1}$ and the prior probability of nodes ' 5 ' and ' 6 ' in $G_{2}$ are $>\rho_{1}$, and their prior probability in $G$ is $\leq \rho_{1}$, these nodes are exposed and Exposed $=<4^{6},{ }^{\prime} 5^{\prime}, 6^{\prime}$ '> $>$.

Out-phase: Consider node ' 4 ' first. There is no safe-move out of $G_{1}$ for links $(1,4)$ and $(5,4)$ because their source nodes have out-degree of 1 . Consider node ' 5 '. Similarly, there is no safe-move out of $G_{2}$ for links $(4,5)$ and $(7,5)$ because their source nodes have out-degree of 1 . Consider node ' 6 '. We can safe-move the link $(5,6)$ from $G_{2}$ to $G_{1}$. Since $\alpha_{2}\left({ }^{\prime} 6^{\prime}\right)=1$, it suffices to move one link, so node ' 6 ' is removed from Exposed.

In-phase: Consider node ' 4 '. Since all links in $G_{2}$ have a source node with out-degree of 1 , there is no safe-move of any link from $G_{2}$ into $G_{1}$. Consider node ' 5 '. We can safemove the link $(2,1)$ from $G_{1}$ to $G_{2}$ to increase $\left|\operatorname{Src}\left(G_{2}\right)\right|$. Since $\beta_{2}\left({ }^{\prime} 5\right.$ ' $)=1$, node ' 5 ' is removed from Exposed.

In the second iteration, Exposed $\left.=<^{\prime} 4^{\prime}\right\rangle$. We can safemove out $(5,4)$ from $G_{1}$ to $G_{2}$. Since $\alpha_{1}\left({ }^{\prime} 4\right.$ ') $=1$, node ' 4 ' is removed from Exposed. There is no more exposed node. The final two subgraphs have the links $E_{1}=\{(4,2),(2,3)$, $(1,4),(5,6)\}$ and $E_{2}=\{(4,5),(5,4),(7,5),(3,6),(5,7),(2,1)\}$.

Remark 3. Algorithm 2 provides no guarantee to eliminate all exposed nodes. In this sense, subgraph-wise perturbation serves a trade-off between utility and privacy. As discussed in Remark 2, social networks are highly sparse and follow a long-tail degree distribution. Thus, we expect that only nodes with a very high in-degree are likely to exceed $\rho_{1}$ after the linking-partitioning, but such nodes are naturally less sensitive because they are commonly referenced. Furthermore, as discussed in Remark 1, for many social network applications, it suffices to publish all $G_{t}^{*}$ in a single merged graph $G^{*}$ without separating them apart. In this case, the prior probability of destination nodes remains unchanged, so the degree balancing step in Algorithm 1 is not needed.

\subsection{Anonymizing Singular Links (Line 5)}

While the problem in Section 5.2 is caused by a large indegree of destination nodes in a subgraph, we now consider a problem caused by a large out-degree of source nodes in a subgraph. Consider $E_{2}$ in Figure 2 where node ' 5 ' is connected to all nodes in $\operatorname{Dst}\left(G_{2}\right)$ but itself. Suppose that the link $(5,7)$ is perturbed to $(5,5)$ or $(5,6)$, we have either a selfloop or duplicate links. Such links are called singular links. Since singular links do not appear in the original graph and since node ' 7 ' is the only unused destination node in $G_{2}^{*}$, it is immediate that the self-loop or the multiple link must come from the original link $(5,7)$.

In general, if a source node $x$ is connected to all or most destination nodes in $G_{t}$ (for a large $G_{t}$, this is very unlikely), there is an increased chance to infer the true destination of a singular link originating at $x$ since each singular link will leave exactly one destination node to which $x$ has no link in $G_{t}^{*}$. This node in fact is the true destination node for the singular link.

Simply discarding the singular links from the perturbed subgraph $G_{t}^{*}$ will interfere with the perturbation intended for providing $\left(\rho_{1}, \rho_{2}\right)$-privacy. Our solution is to keep all singular links in $G_{t}^{*}$ but remove some links from $G_{t}$ prior to the perturbation of $G_{t}$. Specifically, we want to ensure that each source node in $\operatorname{Src}\left(G_{t}\right)$ has no link to some minimum number, say $\ell-1$, of nodes in $\operatorname{Dst}\left(G_{t}\right)$. This condition implies that for a singular link in $G_{t}^{*}$, there are at least $\ell$ choices for its true destination node, thus, bounding the guessing probability by $\frac{1}{\ell}$. 
Definition 5. ( $\ell$-sparsity): Let $\operatorname{Dst}\left(G_{t}\right) \backslash x$ denote $\operatorname{Dst}\left(G_{t}\right)$ without node $x . A$ subgraph $G_{t}$ is $\ell$-sparse if each source node $x \in \operatorname{Src}\left(G_{t}\right)$ is not connected to at least $\ell-1$ destination nodes in $D s t\left(G_{t}\right) \backslash x$. In other words, the out-degree of any node in $\operatorname{Src}\left(G_{t}\right)$ is at most $\left|\operatorname{Dst}\left(G_{t}\right) \backslash x\right|+1-\ell$. $\square$

LEMma 1. If a subgraph $G_{t}$ is $\ell$-sparse, the probability of guessing the true destination node for each singular link in $G_{t}^{*}$ is no more than $\frac{1}{\ell}$.

$\ell$-sparsity with a value of $\ell$ from 5 to 10 should provide sufficient uncertainty. Given that a social network graph is typically large and sparse, we expect that $\ell$-sparsity typically holds for a subgraph $G_{t}$ without further modification to $G_{t}$, unless $G_{t}$ is very small. In the rare case that $G_{t}$ is not $\ell$-sparse, some node $x$ in $\operatorname{Src}\left(G_{t}\right)$ has an out-degree more than $\left|\operatorname{Dst}\left(G_{t}\right) \backslash x\right|+1-\ell$. We can satisfy the condition of $\ell-$ sparsity by deleting no more than $\ell-1$ out-going links $(x, v)$ from $G_{t}$. If $v$ becomes isolated after deleting the link $(x, v)$, $\operatorname{Dst}\left(G_{t}\right)$ still contains $v$. Note that this link removal does not reduce $\left|\operatorname{Src}\left(G_{t}\right)\right|$ because $x$ remains a source for other links, thus, does not increase the prior probability of any node in $G_{t}$ and does not create new exposed nodes. The step $\ell$-sparsity $\left(G_{1}, \ldots, G_{k}, \ell\right)$ in Algorithm 1 is based on this idea.

\section{EXPERIMENTS}

We implemented the proposed algorithms to evaluate the utility of perturbed graphs on a system with core-2 Duo 2.99GHz CPU with 3.83 GB memory. The implementation was done in $\mathrm{VC}++$. For social network analysis we used UCINET software [7].

\subsection{Experiment Setup}

\subsubsection{Data sets}

Our first data set is the e-mail network of University Rovirai Virgili (URV) with 1,132 nodes and 10,900 links [12]. This graph shows e-mail interchanges between members of the university. Data providers eliminated e-mails that were sent to more than 50 different recipients (spam ignored) and also only considered bidirectional interchanges.

The second data set is the Newman's scientific co-authorship network [16] with 16,264 nodes and 95,188 links (47,594 bidirectional links), which is a co-authorship network of scientists posting preprints on the High-Energy Theory in arXiv E-Print Archive between 1995 and 1999. Figure 4 shows the long-tail degree distribution of these data sets.

\subsubsection{Parameters}

For $\left(\rho_{1}, \rho_{2}\right)$-privacy, we set $\rho_{1}$ to 0.01 , and $\rho_{2}$ to $0.1,0.2$, 0.3 , and 0.4 . We set the number of partitions $k$ to 1,10 , 50 and 100 for the $U R V$ network, and to 1, 100, 500, and 1000 for the Newman's network. For both data sets, the generated subgraphs are $\ell$-sparse for $\ell=5$ and all settings of $k$. Therefore, the step $\ell$-sparsity $\left(G_{1}, \ldots, G_{k}, \ell\right)$ in Algorithm 1 can be skipped.

\subsubsection{Evaluation methods}

We compare the following approaches:

Subgraph-Wise Perturbation, with and without degree balancing. This is the proposed Algorithm 1.

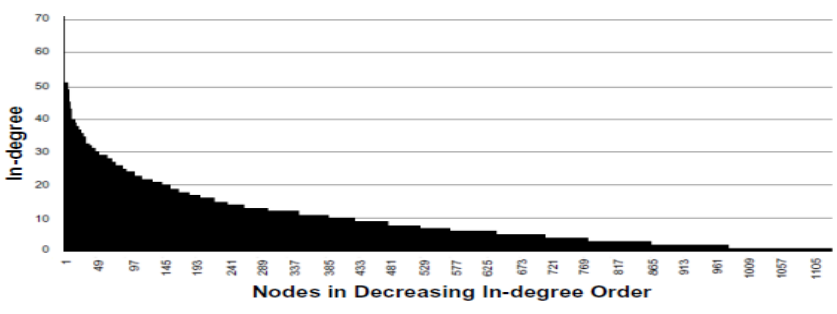

(a) $U R V$ email network

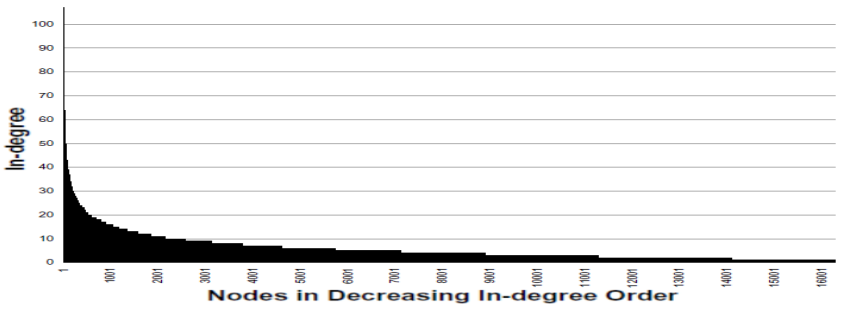

(b) Newman's co-authorship network

Figure 4: Network degree distribution

Table 1: Average values for the $U R V$ email network

\begin{tabular}{|c|c|c|c|c|}
\hline$k$ & 1 & 10 & 50 & 100 \\
\hline$\left|E_{i}\right|$ & 10900 & 1090 & 218 & 109 \\
\hline $\mid$ Dst $\left(G_{i}\right) \mid$ & 1132 & 113.19 & 22.63 & 11.32 \\
\hline $\mid$ Dst $\left(G_{i}\right) \mid($ balancing $)$ & 1132 & 291.39 & 60.41 & 26.21 \\
\hline $\mid$ Src $\left(G_{i}\right) \mid$ & 1132 & 320 & 111.1 & 70.25 \\
\hline $\mid$ Src $\left(G_{i}\right) \mid($ balancing $)$ & 1132 & 466.2 & 142.08 & 81.83 \\
\hline$P_{i i}$ & 0.05 & 0.37 & 0.75 & 0.85 \\
\hline$P_{i i}$ (balancing) & 0.05 & 0.23 & 0.67 & 0.83 \\
\hline Coverage & $100 \%$ & $41.53 \%$ & $11.98 \%$ & $4.85 \%$ \\
\hline Coverage (balancing) & $100 \%$ & $99.75 \%$ & $56.82 \%$ & $41.66 \%$ \\
\hline
\end{tabular}

Table 2: Average values for the Newman's network

\begin{tabular}{|c|c|c|c|c|}
\hline$k$ & 1 & 100 & 500 & 1000 \\
\hline$\left|E_{i}\right|$ & 95188 & 951.88 & 190.37 & 95.188 \\
\hline $\mid$ Dst $\left(G_{i}\right) \mid$ & 16264 & 162.63 & 32.52 & 16.26 \\
\hline $\mid$ Dst $\left(G_{i}\right) \mid($ balancing $)$ & 16264 & 269.70 & 43.38 & 21.30 \\
\hline $\mid$ Src $\left(G_{i}\right) \mid$ & 16264 & 272.02 & 68.56 & 40.56 \\
\hline $\mid$ Src $\left(G_{i}\right) \mid($ balancing $)$ & 16264 & 387.62 & 95.01 & 53.39 \\
\hline$P_{i i}$ & 0.004 & 0.29 & 0.67 & 0.83 \\
\hline$P_{i i}$ (balancing) & 0.004 & 0.28 & 0.65 & 0.81 \\
\hline Coverage & $100 \%$ & $35.65 \%$ & $3.42 \%$ & $0.4 \%$ \\
\hline Coverage (balancing) & $100 \%$ & $90.02 \%$ & $64.52 \%$ & $58.60 \%$ \\
\hline
\end{tabular}

Graph-Wise Perturbation: This is the special case of Subgraph-Wise Perturbation when $k=1$.

Random Add/Del: This is the implementation of the link perturbation approach in [18], which randomly adds $n$ non-existing links and randomly deletes $n$ existing links. $n$ is derived from the method in [19] as follows. Let $A$ and $A^{*}$ be the adjacency matrix of $G$ and $G^{*}$ respectively. According to [19], both the link retention probability and the posterior belief $\operatorname{Pr}\left[a_{i j}=1 \mid a_{i j}^{*}=1\right]$ are equal to $\frac{|E|-n}{|E|}$. We bound $\operatorname{Pr}\left[a_{i j}=1 \mid a_{i j}^{*}=1\right]$ by $\rho_{2}$. Thus $n=\left(1-\rho_{2}\right) \times|E|$. 
We investigate the following utility metrics.

- Link retention probability. For Subgraph-Wise Perturbation and Graph-Wise Perturbation, this is $P_{i i}$, and for Random Add/Del, this is $\frac{|E|-n}{|E|}$, where $n$ is the number of links deleted.

- Reconstruction error. This is the reconstruction error of in-degree distribution in Definition 2. Only Graph-Wise Perturbation and Subgraph-Wise Perturbation use this metric.

- Common graph metrics. We study social network analysis metrics including degree/closeness/betweenness centralities, clustering coefficient, graph diameter, and largest eigenvalue. For detailed definitions, see [17]. These metrics apply to all methods.

\subsection{Results}

\subsubsection{The number of exposed nodes}

First, we examine the impact of Subgraph-Wise Perturbation on the number of exposed nodes, i.e., the destination nodes that have the prior $\leq \rho_{1}$ in $G$ but have the prior $>\rho_{1}$ in some subgraph $G_{t}$. This can be measured by the percentage of destination nodes that have the prior $\leq \rho_{1}$ in all subgraphs $G_{t}$ after graph partitioning, among those destination nodes that have the prior $\leq \rho_{1}$ in $G$. With our setting of $\rho_{1}=0.01$, before graph partitioning, $73 \%$ of destination nodes in the $U R V$ networks have the prior $\leq \rho_{1}$, and $100 \%$ of destination nodes in the Newman's network have the prior probability $\leq \rho_{1}$. The last two rows in Tables 1 and 2 show the percentage of destination nodes that have the prior $\leq \rho_{1}$ in all subgraphs $G_{t}$, with and without degree balancing, among the destination nodes that have the prior $\leq \rho_{1}$ in $G$.

With a larger number of subgraphs, $k,\left|\operatorname{Src}\left(G_{t}\right)\right|$ for each subgraph $G_{t}$ becomes smaller, thus, more nodes become exposed after graph partitioning. In this case, degree balancing is effective to reduce the number of exposed nodes. For example, with $k=10$ for $U R V$ and $k=100$ for Newman, without degree balancing, $41.53 \%$ and $35.65 \%$ of those with the prior $\leq \rho_{1}$ in $G$ also have $\leq \rho_{1}$ in all subgraphs $G_{t}$, but with degree balancing, these percentages are $99.75 \%$ and $90.02 \%$, respectively. In other words, except for a very large $k$, most nodes that have their posterior bounded by $\rho_{2}$ in $G^{*}$ also have their posterior bounded by $\rho_{2}$ in all subgraphs $G_{t}^{*}$

\subsubsection{Link retention probability}

Retention probability is a direct indicator of how much original links are preserved. For Subgraph-Wise Perturbation, the left side of Figure 5 shows the retention probability vs $\rho_{2}$ and the number of subgraphs $k$. The retention probability increases as $\rho_{2}$ increases, and a larger $k$ results in a significant higher retention probability. In fact, a larger $k$ implies that the domain size $\left|\operatorname{Dst}\left(G_{t}\right)\right|$ for destination nodes in a subgraph $G_{t}$ is reduced, and according to Equation (4), this contributes to a larger retention probability. Tables 1 and 2 show the detailed statistics on $\left|E_{t}\right|,\left|D s t\left(G_{t}\right)\right|$, $\left|\operatorname{Src}\left(G_{t}\right)\right|$, and retention probability $P_{i i}$, with respect to $k$. $\rho_{1}$ is set to 0.01 and $\rho_{2}$ is set to 0.4 .

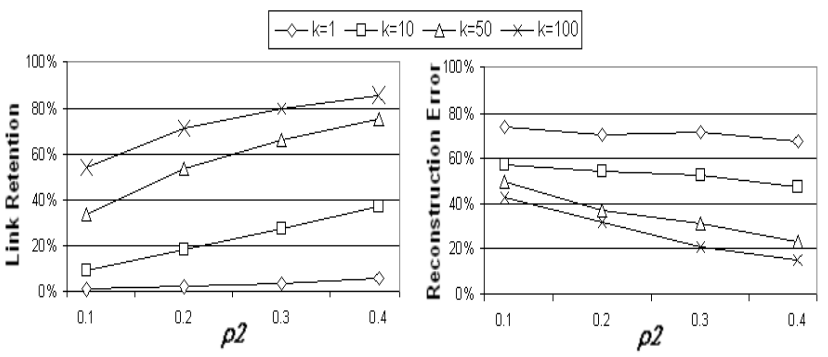

(a) $U R V$ email network - without degree balancing

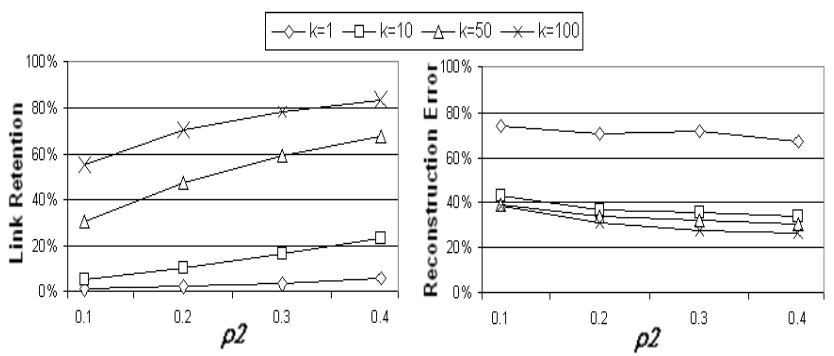

(b) $U R V$ email network - with degree balancing

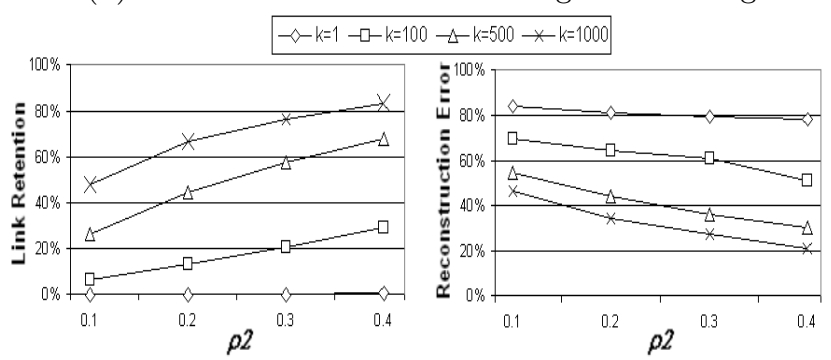

(c) Newman's co-authorship network - without degree balancing

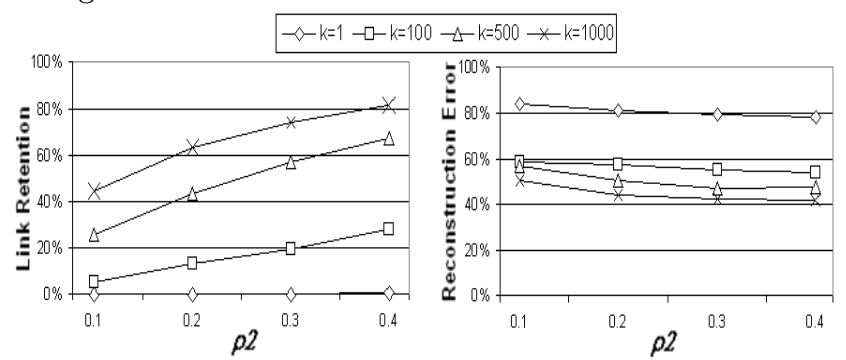

(d) Newman's co-authorship network - with degree balancing

Figure 5: Link retention and reconstruction error comparison

The link retention probability for Random Add/Del is equal to $\frac{|E|-n}{|E|}$, which is bounded by $\rho_{2}$, that is, $0.1,0.2$, 0.3 and 0.4. These probabilities are lower than those of Subgraph-Wise Perturbation for a large $k$. This study supports our claim that subgraph-wise perturbation better retains structural information than graph-wise perturbation.

\subsubsection{In-degree reconstruction error}

This experiment studies the accuracy of reconstructing the in-degree of each node from the published graph, where the accuracy is measured by the reconstruction error (Definition 2). The right side of Figure 5 shows the reconstruction error of in-degree distribution vs $\rho_{2}$ and $k$, where $k$ is 


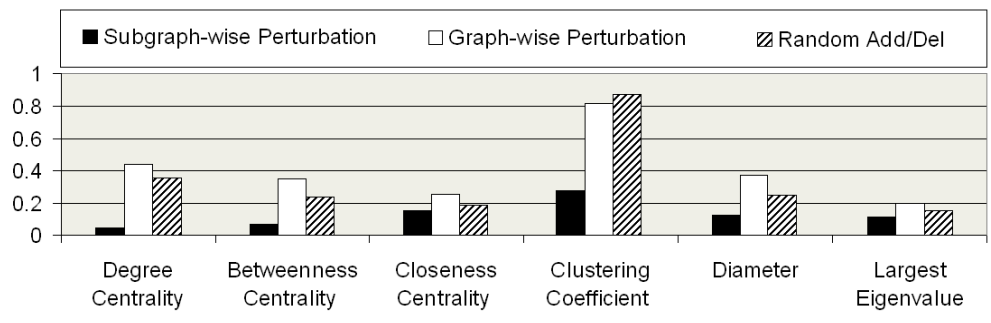

(a) $U R V$ email network

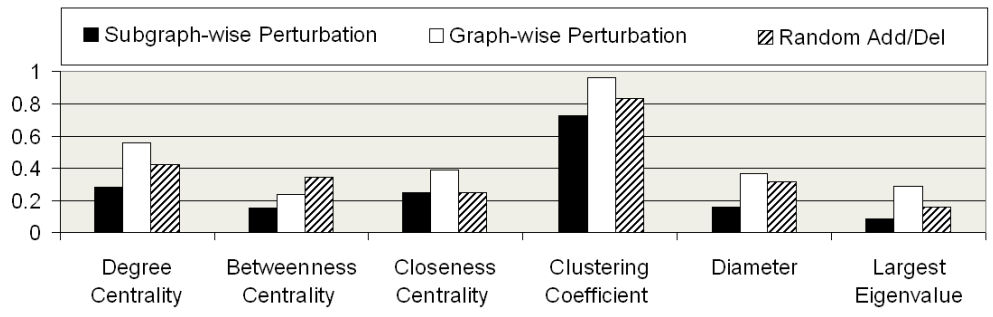

(b) Newman's co-authorship network

Figure 6: Relative error for social network analysis

the number of partitions. Without degree balancing, for a larger $k$, the increased retention probability contributes to a decreased reconstruction error. With degree balancing, the effect of increasing $k$ to reduce the reconstruction error is less significant. This is because degree balancing increases $m=\left|\operatorname{Dst}\left(G_{t}\right)\right|$ in a receiving subgraph $G_{t}$ of a link, thus, reduces the retention probability in $G_{t}$ according to Equation (4).

This study suggests that subgraph-wise perturbation is more effective in reducing the reconstruction error when protecting low in-degree nodes is the priority, in which case degree balancing is not needed.

\subsubsection{Social network analysis}

One important motivation of publishing social network data is to study a variety of social network behaviors. Figure 6 shows the relative error of four methods for common social network metrics. Subgraph-wise perturbation is run without the degree balancing step because we only publish a single merged graph $G^{*}$ for performing social network analysis, as discussed in Remarks 1 and 3. For each method, we run 10 trials of randomization and reported their average results. The values of closeness/degree/betweenness centralities are the average over all nodes. In this experiment, $\rho_{1}$ is set to the default value, $\rho_{2}$ is set to 0.4 , and for Subgraph-Wise Perturbation, $k=100$ for the email network and $k=1000$ for the co-authorship network.

For almost the metrics studied, Subgraph-Wise Perturbation gives more accurate results than Graph-Wise Perturbation and Random Add/Del. Graph-Wise Perturbation has a large error across most metrics used. Indeed, all these metrics depend on the local structure (such as degree centrality and clustering coefficient) and/or global structure (such as diameter and largest eigenvalue) of the graph to a varied degree. Compared to Graph-Wise Perturbation, SubgraphWise Perturbation better preserves such structures by retaining more original destination nodes of links and replacing an original destination node with a structurally close destination node when a link is perturbed. This study supports our claim that Subgraph-Wise Perturbation is superior to Graph-Wise Perturbation for general social network data analysis.

\section{CONCLUSION}

We presented a novel link perturbation algorithm, subgraphwise perturbation, to limit link disclosure in publishing social network data. This algorithm addresses the excessive structural distortion in previous link perturbation methods through two novel modifications: It treats a social network as a directed graph and perturbs only the destination of a link, instead of both the source and the destination, and it partitions a large graph into several small subgraphs and perturbs the destination nodes within each subgraph. Compared to the previous graph-wise perturbation, subgraphwise perturbation is able to preserve more graph structure while providing a given privacy guarantee by (1) completely preserving the source nodes of all links, (2) retaining more original destination nodes of links with a larger retention probability, and (3) confining the randomized destination node of a link to a local neighborhood. Additionally, we also factor the popularity of destination nodes through a privacy notion of a destination node relative to this popularity. To our best knowledge, this is the first work that preserves structural properties through link destination perturbation within a local subgraph and factors the popularity of destination nodes in privacy of such nodes.

\section{ACKNOWLEDGMENT}

The authors would like to thank reviewers of EDBT 2012 conference for their feedback. The work of Ke Wang was supported by Canada NSERC Discovery Grant, and the work of Philip S. Yu was supported by US NSF through grants CNS-1115234, and IIS-0914934, and Google Mobile 2014 Program. 


\section{REFERENCES}

[1] C. C. Aggarwal, and P. S. Yu. Privacy-preserving data mining: models and algorithms. Vol. 34, Advances in Database Systems, Springer, 2008

[2] R. Agrawal, R. Srikant, and D. Thomas. Privacy preserving OLAP. In SIGMOD 2005

[3] L. Backstrom, C. Dwork, and J. M. Kleinberg. Wherefore art thou R3579X?: anonymized social networks, hidden patterns, and structural steganography. In WWW, 2007

[4] K. Bai, Y. Liu, and P. Liu. Prevent identity disclosure in social network data study. In ACM CCS 2009

[5] A.-L. Barabasi, R. Albert. Emergence of scaling in random networks. In Science, Vol 286, 509-512, 1999

[6] F. Bonchi, A. Gionis, and T. Tassa. Identity obfuscation in graphs through the information theoretic lens. In ICDE 2011.

[7] S.P. Borgatti, M.G. Everett, and L.C. Freeman. Ucinet for windows: software for social network analysis. Harvard, MA: Analytic Technologies 2002.

[8] R. Chaytor, and K. Wang. Small domain randomization: same privacy, more utility. In VLDB 2010

[9] J. Cheng, A. W. Fu, and J. Liu. K-isomorphism: privacy preserving network publication against structural attacks. In SIGMOD 2010

[10] A. Evfimievski, J. Gehrke, and R. Srikant. Limiting privacy breaches in privacy preserving data mining. In PODS 2003

[11] B. C. M. Fung, K. Wang, A. W.-C. Fu, and P. S. Yu. Introduction to privacy-preserving data publishing: concepts and techniques. Data Mining and Knowledge Discovery Series. Chapman \& Hall/CRC, 2010

[12] R. Guimera, L. Danon, A. Diaz-Guilera, F. Giralt and A. Arenas. Self-similar community structure in a network of human interactions. In Physical Review E, Vol. 68, 2003

[13] M. Hay, G. Miklau, D. Jensen, P. Weis, and S. Srivastava. Anonymizing social networks. Technical report, University of Massachusetts Amherst, 2007

[14] G. Karypis, V. Kumar. A fast and high quality multilevel scheme for partitioning irregular graphs. In SIAM Journal on Scientific Computing, Vol. 20, 1999

[15] K. Liu, and E. Terzi. Towards identity anonymization on graphs. In ACM SIGMOD/PODS 2008

[16] M. E. J., Newman. The structure of scientific collaboration networks. In Proc. of the National Academy of Sciences of the USA, Vol. 98, No. 2. 404-409. 2001.

[17] S. Wasserman and K. Faust. Social network analysis: methods and applications. Cambridge University Press, 1994

[18] X. Ying, and X. Wu. Randomizing social networks: a spectrum preserving approach. In SDM 2008

[19] X. Ying and X. Wu. On link privacy in randomizing social networks. In PAKDD 2009

[20] L. Zhang, and W. Zhang. Edge anonymity in social network graphs. In IEEE Social Computing 2009

[21] E. Zheleva, and L. Getoor. Preserving the privacy of sensitive relationships in graph data. In PinKDD 2007

[22] E. Zheleva, and L. Getoor. To join or not to join: the illusion of privacy in social networks with mixed public and private user profiles. In WWW 2009

[23] B. Zhou and J. Pei. Preserving privacy in social networks against neighborhood attacks. In ICDE 2008

[24] L. Zou, L. Chen, and M. T. Ozsu. K-automorphism: a general framework for privacy preserving network publication. In VLDB 2009. 